\title{
Neoplasia Folicular da Tiroide Não Invasiva com Aspetos Nucleares de Carcinoma Papilífero
}

\section{Noninvasive Follicular Thyroid Neoplasm with Papillary-Like Features}

Paulo Costa Correia ${ }^{1,2}$, Liliana Coutinho1,2, Pedro Caldes ${ }^{2}$

\section{RESUMO}

A neoplasia folicular da tiroide não invasiva com aspetos nucleares de carcinoma papilar (NIFTP) é uma neoplasia encapsulada com padrão de crescimento folicular e características nucleares do carcinoma papilar da tiroide. Eram conhecidos como "variante folicular não-invasiva e encapsulada do carcinoma papilar da tiroide, mas dada a sua semelhança biológica aos adenomas foliculares (comportamento indolente e ausência de metástases linfáticas ou recorrência), a nomenclatura foi alterada. Em 2015 um painel de especialistas decidiu chamar-Ihe NIFTP. Apesar da elevada suspeição, o diagnóstico definitivo apenas é possível através do estudo histológico da peça cirúrgica. Uma vez feito o diagnóstico, não sendo uma lesão maligna, não é necessário submeter os doentes a estadiamento nem protocolos de tratamento oncológico.

Apresentam-se dois casos clínicos em que se procedeu a tiroidectomia total em contexto multinodular. A NIFTP é muitas vezes tratada excessiva/agressivamente, o que acarreta morbilidade e stress psicológico para os doentes, bem como custos desnecessários aos sistemas de saúde.

PALAVRAS-CHAVE: Adenocarcinoma Folicular; Carcinoma Papilífero da Tiroide; Neoplasias da Tiroide 


\begin{abstract}
Noninvasive thyroid follicular neoplasia with nuclear aspects of papillary carcinoma (NIFTP) is an encapsulated neoplasm with follicular growth pattern and nuclear features of papillary thyroid carcinoma. They were known as "noninvasive and encapsulated follicular variant of papillary thyroid carcinoma, but given their biological similarity to follicular adenomas (indolent behavior and absence of lymphatic metastases or recurrence), the nomenclature was altered. In 2015 a panel of experts decided to call it NIFTP. Despite the high suspicion, the definitive diagnosis is only possible through the histological study of the surgical specimen. Once the diagnosis is made, not being a malignant lesion, it is not necessary to subject patients to staging or cancer treatment protocols.

Two clinical cases were presented in which total thyroidectomy was performed in a multinodular context.

NIFTP is often overtreated/aggressively which causes morbidity and stress.
\end{abstract}

KEYWORDS: Adenocarcinoma, Follicular; Carcinoma, Papillary; Thyroid Neoplasms

\section{INTRODUÇÃO}

As neoplasias malignas da tiroide são as mais frequentes do sistema endócrino. Nas últimas três décadas assistiu-se a um aumento significativo da incidência deste tipo de tumores (fundamentalmente à custa dos carcinomas diferenciados da tiroide (CDT), dos carcinomas papilares em particular). Em 2012 as taxas de incidência no género feminino eram aproximadamente três vezes superiores às verificadas no género masculino (9,3 e 3,1 casos por 100000 pessoas - ano respetivamente). Não obstante a taxa de mortalidade continua baixa (0,7 e 0,5 casos por 100000 pessoas - ano para mulheres e homens respetivamente). ${ }^{1}$

Classificam-se de acordo com o seu tipo histológico e a maioria dos tumores tiroideus com exceção do carcinoma medular, deriva da célula folicular, que origina neoplasias benignas/malignas com diferentes características fenotípicas, biológicas e clínicas. Os carcinomas papilares e foliculares são considerados diferenciados (CDT), uma vez que mantêm uma semelhança estrutural e funcional com o tecido tiroideu normal e são responsáveis por $94 \%$ dos tumores da tiroide. ${ }^{2,3} \mathrm{O}$ comportamento é variável de formas leves a extremamente agressivas de elevada mortalidade, mas regra geral, com prognóstico na maior parte das vezes, favorável. ${ }^{4}$

Os CDT manifestam-se através de um nódulo na tiroide isolado ou num contexto de um bócio multinodular. A punção aspirativa por agulha fina (PAAF) permite classificá-los em seis categorias e correlaciona o resultado com a probabilidade de carcinoma de tiroide $^{5}$ - classificação de Bethesda (I/VI). A maioria dos tumores malignos pode ser identificada por citologia. Os carcinomas foliculares e neoplasias foliculares da tiroide não invasi- va com aspetos nucleares de carcinoma papilar (NIFTP) são exceções, sendo geralmente classificados como indeterminados.

O carcinoma papilar engloba vários tipos histológicos: clássico, encapsulado, folicular, macrofolicular, de células altas, de células colunares, de células claras, carcinoma esclerosante difuso, difuso, cribiforme, oncocítico, microcarcinoma, entre outros. O tumor é multicêntrico em 20\%-80\% dos casos e bilateral em cerca de metade. Recentemente, Shattuck et al ${ }^{6}$ avaliaram se focos contíguos do tumor, surgem como metástases de um tumor primário ou se originam de novos clones com precursores independentes e concluíram, que em tumores com carcinoma multifocal, os tumores papilares são tumores independentes.

O acompanhamento destes pacientes é feito de uma forma geral, com a monitorização de vários parâmetros, com sensibilidades e especificidades diferentes. O tipo de exames e a periodicidade dependem do tipo histológico, tratamento inicial, risco de recidiva/persistência da doença e resposta ao tratamento. $O$ doseamento da tireoglobulina e a ecografia cervical são os pilares do follow-up dos tumores diferenciados da tiroide.

A NIFTP é um tumor não invasivo encapsulado ou clinicamente delimitado, com um padrão de crescimento e características nucleares de carcinoma papilar da tiroide. A ecografia, a citologia e os testes moleculares são úteis para o estudo de nódulos tiroideus, mas há grande sobreposição dos resultados com a variante folicular encapsulada de CPT e outros tumores com padrão folicular. O diagnóstico de NIFTP é realizado após estudo histológico da peça operatória. A cápsula pode ser fibrosa, espessa, fina, mas clinicamente demarcada do tecido 
tiroideu adjacente. $\bigcirc$ padrão de crescimento é folicular e pode ser micro, normo ou macro folicular com coloide, por vezes, abundante. As características nucleares são as do carcinoma papilar-like com núcleos volumosos, alongados, sobrepostos, com irregularidades da membrana nuclear, dando a ilusão de fendas nucleares (pseudo - inclusões), e núcleos vitrais, em vidro fosco ("ground-glass"), com cromatina dispersa, que causa um aparente vazio, em aspeto de "olho de órfã de Annie". Existem também invaginações no citoplasma. 7,8

\section{CRITÉRIOS DE NIKIFOROV - ABRIL DE 2016 ${ }^{8}$ :}

- Encapsulamento ou demarcação clara do tumor do tecido tiroideu adjacente sem nenhuma invasão;

- Padrão de crescimento folicular com menos de 1\% de papilas, ausência de corpos psamomatosos e menos de 30\% de padrão de crescimento sólido, trabecular ou insular);

- Características nucleares de carcinoma papilífero (em grau 2 e 3);

- Ausência de invasão da cápsula;

- Inexistência de necrose;

- Escassa atividade proliferativa (menos de 3 mitoses/ campo).

São sempre critérios de exclusão: invasão da cápsula ou vascular, estruturas papilares verdadeiras em mais de $1 \%$ do volume do tumor, necrose tumoral, qualquer característica celular ou morfologia, de qualquer outra variante de carcinoma papilar, sem corpos psamomatosos ou qualquer mutação dos promotores BRAF v6ooo e TERT, ou qualquer metástase.

A excisão do tumor é suficiente não sendo necessária a totalização da tiroidectomia, ou qualquer dissecção ganglionar cervical, iodoterapia ou terapia supressora.

Descrevemos dois casos clínicos de NIFTP em contexto de tiroides multinodulares.

\section{CASOS CLÍNICOS}

\section{CASO 1}

Paciente do género masculino com 45 anos, que apresentava nódulo da linha média do pescoço de consistência firme, contornos regulares, indolor e móvel.

Os doseamentos hormonais da função da glândula tiroide eram normais e a ecografia demonstrava uma tiroide multinodular com nódulo do istmo, bem delimitado, com
$3 \mathrm{~cm}$. Diversos nódulos em toda a glândula de menores dimensões. PAAF-tumor folicular (Bethesda IV).

Submetido a tiroidectomia total, sem intercorrências.

O estudo histológico evidenciou: no istmo lesão de 2,9 cm correspondendo a um NIFTP-OMS; nódulos microfoliculares na glândula restante (Fig.s 1 e 2).

\section{CASO 2}

Paciente do género feminino com 75 anos com nódulo da região látero-cervical esquerda de consistência firme, indolor e móvel.

Os doseamentos hormonais eram normais e a ecografia mostrava um nódulo de 2,5cm no lobo esquerdo, com vários nódulos de menores dimensões. PAAF-Lesão folicular de natureza indeterminada (Bethesda III).

Submetida a tiroidectomia total sem intercorrências.

O estudo histológico evidenciou: no lobo esquerdo da glândula NIFTP-OMS de $2 \mathrm{~cm}$ em contexto de bócio multinodular adenomatoso (Fig.s 3 e 4).

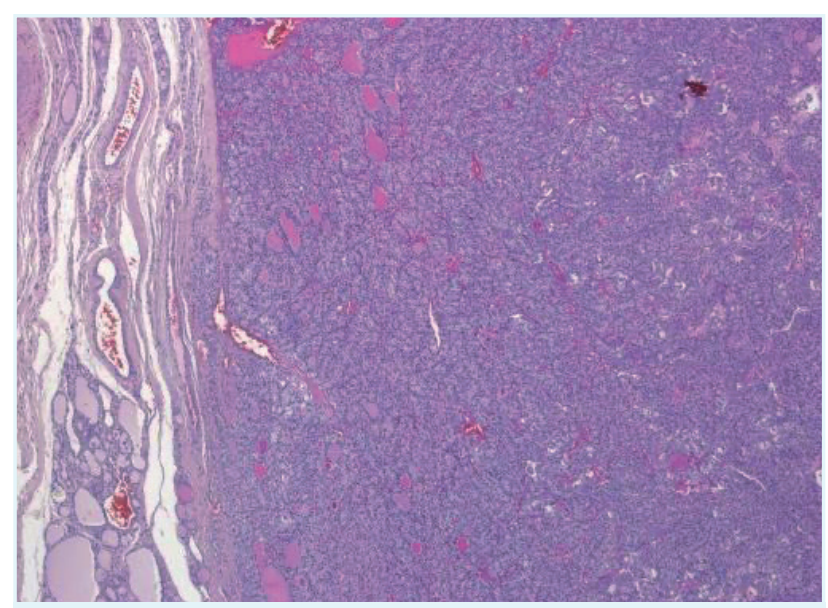

FIGURA 1. Caso 1.

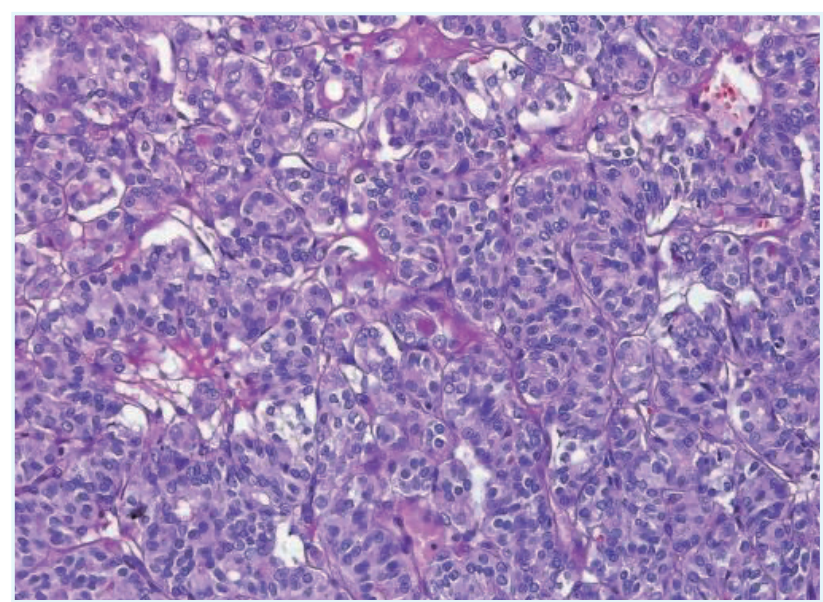

FIGURA 2. Caso 1. 


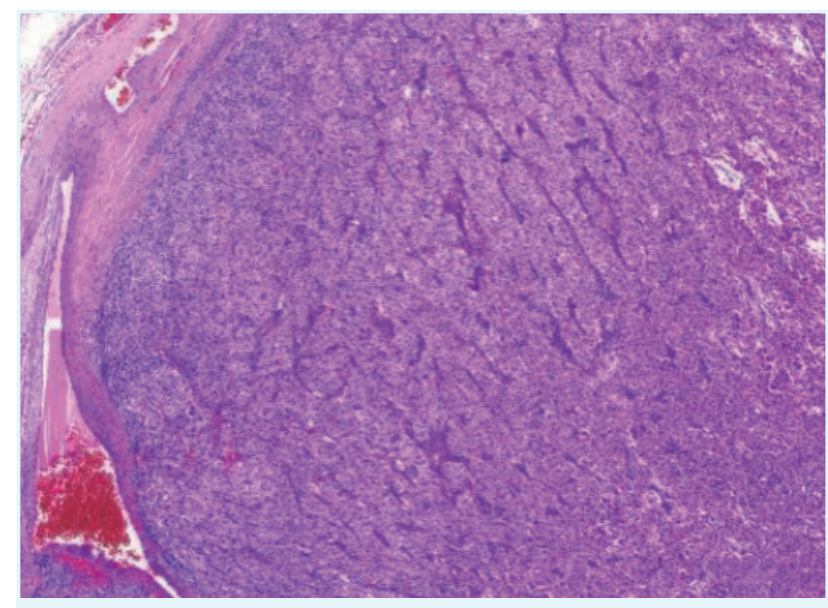

FIGURA 3. Caso 2.

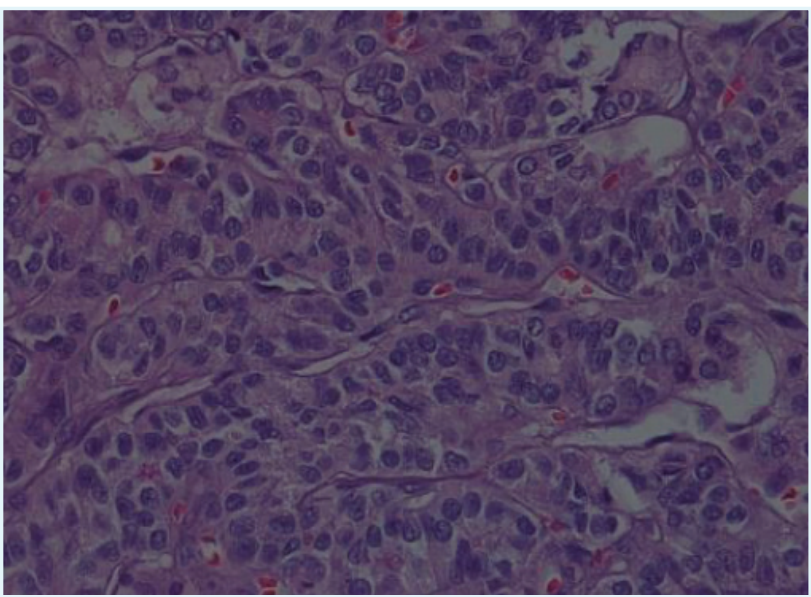

FIGURA 4. Caso 2.

\section{CONCLUSÃO}

A eliminação da designação "carcinoma" da definição desta variante de carcinoma papilar da tiroide enfatiza o excelente prognóstico: não há registo de mortes relacionadas e o risco de recorrência é inferior a 1\%.

O diagnóstico de NIFTP (s) requer um exame anatomopatológico minucioso. A identificação correta reduz o recurso desnecessário a procedimentos cirúrgicos radicais e a administração de iodo radioativo após tiroidectomia. O follow-up é sobreponível aos carcinomas de muito baixo risco.

Apesar de se tratar de NIFTP (s) e de a lobectomia/istmectomia ser uma solução adequada e suficiente nestas neoplasias, nos doentes apresentados optou-se pela tiroidectomia total, pelo facto das lesões se enquadrarem em bócios multinodulares. Os doentes foram dispensados de qualquer outra terapêutica e/ou acompanhamento que não fosse o suplemento com levotiroxina.

Somos de opinião que o reconhecimento desta entidade clínica (NIFTP) venha agora reforçar uma indicação, para uma atitude mais conservadora, isto é, menos invasiva nas citologias tipo III e IV da classificação de Bethesda.

\section{RESPONSABILIDADES ÉTICAS}

CONFLITOS DE INTERESSE: Os autores declaram a inexistência de conflitos de interesse na realização do presente trabalho.

FONTES DE FINANCIAMENTO: Não existiram fontes externas de financiamento para a realização deste artigo.

CONFIDENCIALIDADE DO DADOS: Os autores declaram ter seguido os protocolos da sua instituição acerca da publicação dos dados de doentes.

CONSENTIMENTO: Consentimento do doente para publicação obtido.

PROVENIÊNCIA E REVISÃO POR PARES: Não comissionado; revisão externa por pares.

\section{ETHICAL DISCLOSURES}

CONFLICTS OF INTEREST: The authors have no conflicts of interest to declare.

FINANCING SUPPORT: This work has not received any contribution, grant or scholarship.

CONFIDENTIALITY OF DATA: The authors declare that they have followed the protocols of their work center on the publication of data from patients.

PATIENT CONSENT: Consent for publication was obtained.

PROVENANCE AND PEER REVIEW: Not commissioned; externally peer reviewed.

\section{REFERÊNCIAS}

1. Filetti S, Durante C, Hartl D, Leboulleux S, Locati LD, Newbold $\mathrm{K}$, et al; ESMO Guidelines Committee. Electronic address: clinicalguidelines@esmo.org. Thyroid cancer: ESMO Clinical Practice Guidelines for diagnosis, treatment and follow-up. Ann Oncol. 2019;30:1856-83. doi: 10.1093/annonc/mdz400.

2. Edwards BK, Howe HL, Ries LA, Thun MJ, Rosenberg HM, Yancik R, Wingo PA, et al. Annual report to the nation on the status of cancer, 1973-1999, featuring implications of age and aging on U.S. cancer burden. Cancer. 2002;94:2766-92. doi: 10.1002/cncr.10593.

3. Golbert L, Wajner SM, Rocha AP, Maia AL, Gross JL. Carcinoma diferenciado da tiroide. Avaliação Inicial e Acompanhamento. Arq Bras Endocrinol Metab. 2005;49:701-10.

4. De Grort LJ, Larsen PR, Hennemain G. The Thyroid and its diseases. 6th ed. New York: Churchill Livingstone; 1996.

5. Cibas ES, Ali SZ. The 2017 Bethesda System for Reporting Thyroid Cytopathology. Thyroid. 2017;27:1341-6. doi:10.1089/thy.2017.0500 
6. Shattuck TM, Westra WH, Ladenson PW, Arnold A. Independent clonal origins of distinct tumor foci in multifocal papillary thyroid carcinoma [published correction appears in N Engl ] Med. 2005;353):1640. N Engl J Med. 2005;352:2406-12. doi:10.1056/NEJMoa044190.

7. Rosario PW, Mourão GF. Follow-up of noninvasive follicular thyroid neoplasm with papillary-like nuclear features (NIFTP). Head Neck. 2019;41:833-4. doi:10.1002/hed.25550.

8. Nikiforov YE, Seethala RR, Tallini G, Baloch ZW, Basolo F, Thompson LD, et al. Nomenclature Revision for Encapsulated Follicular Variant of Papillary Thyroid Carcinoma: A Paradigm Shift to Reduce Overtreatment of Indolent Tumors. JAMA Oncol. 2016;2:1023-9. doi: 10.1001/jamaoncol.2016.0386. 